\title{
Chaotic Fractal Tiling for the Missing Dark Energy and Veneziano Model
}

\author{
L. Marek-Crnjac ${ }^{1}$, M. S. El Naschie ${ }^{2}$ \\ ${ }^{1}$ Technical School Center, Maribor, Slovenia \\ ${ }^{2}$ Department of Physics, Faculty of Science, University of Alexandria, Alexandria, Egypt \\ Email: leila.marek@guest.arnes.si,Chaossf@aol.com
}

Received September 5, 2013; revised October 5, 2013; accepted October 12, 2013

Copyright (C) 2013 L. Marek-Crnjac, M. S. El Naschie. This is an open access article distributed under the Creative Commons Attribution License, which permits unrestricted use, distribution, and reproduction in any medium, provided the original work is properly cited.

\begin{abstract}
The formula for the quantum amplitude of the Veneziano dual resonance model is shown to be formally analogous to the dimensionality of a K-theoretical fractal quotient manifold of the non-commutative geometrical type. Subsequently this analogy is used to deduce the ordinary energy of the quantum particle and the dark energy of the quantum wave. The results agree completely with cosmological measurements. Even more surprisingly the sum of both energy expressions turned out to be exactly equal to Einstein's iconic formula $E=m c^{2}$. Consequently Einstein's formula makes no distinction between ordinary and dark energy.
\end{abstract}

Keywords: Hausdorff Dimension; Cantor Set; Dark Energy; Kähler Manifold; Quantum Entanglement; Veneziano Model

\section{Introduction}

We utilize a formal analogy between Veneziano's formula for the amplitude of the dual resonance model and that for the Hausdorff fractal dimensionality of quotient manifold to develop an energy-mass quantum relativity equation which turned to be of the form $E_{Q R}=\lambda E$ where $E=m c^{2}$ is the famous formula of Einstein's special relativity and $\lambda$ is a scaling quasi exponent equal to $\phi^{5} / 2 \cong 1 / 22$ where $\phi^{5}$ is Hardy's quantum entanglement and $\phi=2 /(\sqrt{5}+1)$. We calculate that way the deficit energy density balance of the universe and find that this $95.4915028 \%$ deficit compared with Einstein's $E=m c^{2}$ maximal total energy density is the hypothetical dark energy which is in turn a confirmation not only of the real existence of dark energy but also of the exactness of the cosmological measurements of WMAP and certain supernova analysis by the three 2011 Nobel Laureates. More importantly the analysis clearly shows the inadequacy of the traditional interpretation of the energy-mass relationship of special relativity when applied to the problem of immensely large distance scales of the order of magnitude of Hubble radius. The correct way to understand $E=m c^{2}$ is to realize that only $E(O) \cong m c^{2} / 22$ is ordinary energy, which can be measured and corresponds to the energy of the quantum particle. The rest, i.e. $E(D) \cong(21 / 22) m c^{2}$ is the dark energy of the quantum wave and cannot be measured in a direct way because of quantum wave collapse. It is gratifying to note a new result not realized in our earlier paper, namely that the sum is $E(O)+E(D)=E($ Einstein $)$ and that chaotic dynamics and fractal geometry lead to an elegant unified picture for nature [1-12].

\section{Background Information and Preliminary Remarks}

There is a serious well documented huge discrepancy between cosmological measurements and theory with regard to the total energy content of the cosmos [1-4]. This was brought about when cosmologists discovered around 1998 that the universe is not only expanding but that its expansion is accelerating [1,2]. This is in clear contradiction to our classical understanding of gravity which says that attraction between mass in the universe should cause the expansion to slow down [1-4]. The deviation of theory from reality is not two or even ten percent but rather a staggering $95.5 \%$ of the energy of the universe is presumed to be missing [1]. This totally unexpected result of the most profound problem in physics 
and cosmology lead to postulating a hypothetical repulsive force called dark energy to explain the accelerated expansion of the universe [1-6]. We trace this discrepancy back to wrongly interpreting special relativity $E=m c^{2}$ [3-6]. Thus in this paper we will be concerned with revising the interpretation of $E=m c^{2}$ where $E$ is the energy, $m$ is the mass and $c$ is the velocity of light by including quantum particle physics [7-11] as well as quantum entanglement [12], topological quantum field theory [13], effective quantum gravity [14] and superstrings $[15,16]$ in our deterministic chaos-fractal analysis [17-28].

There are many ways to show that the correct quantum relativity [27,28] $E_{Q R}$ must be a simple scaling [4,5,23] of $E$ so that $E_{Q R}=\lambda E$ where $\lambda$ is the scaling exponent [4-6]. It turns out that Veneziano's model of dual resonance is one of numerous other ways to handle the problem but has the advantage of being relatively conservative and familiar way $[7,8]$. At the end we find that $\lambda=1 / 22$ where the 22 are Veneziano's 26 dimensions needed for getting rid of negative norms [7-11] from his theory after substituting the four dimensionality of space-time so that $26-4=22$ and $E=m c^{2} / 22$ [27]. Explaining how to do this and distinguishing between ordinary energy and dark energy is our main concern in what follows [22-28].

\section{The Veneziano Amplitude and the Dimensionality of Quotient Manifolds}

Based on previous work by Regge and others, Veneziano [7-10] was able to write down an amplitude for the dispersion scattering of four particles according to his dual resonance model. In conventional notation this is [7,9]

$$
A(a, b)=\int_{0}^{1} x^{a-1}(1-x)^{b-1} \mathrm{~d} x=\frac{\Gamma(a) \Gamma(b)}{\Gamma(a+b)} .
$$

Here $a$ and $b$ are parameters depending upon the momentum of colliding particles, the function $x^{a}$ is real line multiplicative character and the gamma function involved is labeled $\Gamma(\ldots .$.$) [9]. Suppose we can set$ $\Gamma(a)=a, \quad \Gamma(b)=b$ and $\Gamma(a+b)=a+b$, then there will be a formal analogy between $A(a, b)$ and the inverse of the dimension of a quantum manifold of the $X$ space type used in non-commutative geometry [10]

$$
D=\frac{a+b}{(a)(b)}
$$

where $a$ and $b$ here are the dimensions of two sub-manifolds forming an $X$ quotient manifold such as Penrose fractal tiling [11]. Since $D$ is either a Hausdorff or topological dimension, the $1 / D$ is a normed probability and can be compared directly with $A(a, b)$ of Veneziano [7,9]. That means $A(a, b)=1 / D(a, b)$.
The above is naturally part and parcel of the Bosonic Nambu-Goto [16] string which will not be explained here but we will touch upon this point again later on.

\section{Calculating the Topological and Hausdorff Dimensions as Well as Probabilities}

Let us use $D$ to derive first the topological dimension and second the Hausdorff dimension of a Hilbert $4 D$ hypercube $[17,18]$. In the first case we just need to set $a=b=$ $1 / 2$ and find

$$
D_{T}=\frac{(1 / 2) \oplus(1 / 2)}{(1 / 2) \otimes(1 / 2)}=1 /(1 / 4)=4 .
$$

In the second case we have $a=\phi, \quad b=1-\phi=\phi^{2}$ where $\phi=\frac{2}{\sqrt{5}+1}$ and one finds $[10,17,18]$

$$
D_{H}(\text { Hilbert })=\frac{\phi+\phi^{2}}{(\phi)\left(\phi^{2}\right)}=\frac{1}{\phi^{3}} .
$$

In terms of a continued fraction expansion of $\frac{1}{\phi^{3}}$ this is

$$
D_{H}=4+\frac{1}{4+\frac{1}{4+\frac{1}{4+\cdots}}}=4+\phi^{3}=4.23606799 .
$$

Needless to mention that the same result is found from the inverse value of Veneziano's amplitude formula [79]. It was not long before trouble came into the dual resonance model in the form of ghosts or negative norms, i.e. negative quasi probabilities $[8,9,15]$ which made this tangible simple model unreal. To get rid of negative norms, the price was to assume that the model is embedded in 26 dimensions [18]. Thus we either accept ghosts or reconcile the anomalies via 26 dimensions. It seems that Veneziano reluctantly accepted the 26 dimensional space which was the beginning of modern string theory [15] while convinced that it will turn out to be just a mathematical neat trick and no one will ever worry about seeing the invisible $26-4=22$ dimensions. Now with hindsight and in retrospect, if dark energy really exists and we believe that it exists because of its qualitative and quantitative consistency with the increased rate of acceleration of the expansion of the cosmos, then it is hidden in the 22 extra dimensions of Veneziano-Nambu-Goto Bosonic string theory $[7-9,16]$ as we will reason shortly. To show that we consider first a hyper Hilbert cube $[17,18]$ not in 4 but in 11 dimensions. 


\section{An Eleven Dimensional M-Theory Hyper Hilbert-Fractal Cube}

In analogy with $D=4+\overline{4}$ we now construct $D=$ $11+\overline{11}$. This basically gives us a Hausdorff dimension

$$
\begin{aligned}
& D(11)=11+\frac{1}{11+\frac{1}{11+\frac{1}{11+\cdots}}}=11+\phi^{5} \\
& =11.09016994393=11+(\mathrm{k} / 2)
\end{aligned}
$$

where $k=\phi^{3}\left(1-\phi^{3}\right)=0.18033989$ and $\phi=(\sqrt{5}-1) / 2=0.618033989$. This is in effect an 11 dimensional cube inside another 11 dimensional cube and so on like an infinite Russian doll $[17,18]$. Now the probability corresponding to $D(11)$ which we call a fractal M-theory space-time for obvious reasons, is simply the inverse value which due to nice number theoretical properties of $\phi=(\sqrt{5}-1) / 2$

$$
P\left(11+\phi^{5}\right)=\frac{1}{11+\phi^{5}}=\phi^{5}=k / 2=0.090169945 \text {. }
$$

Actually this $\phi^{5}=k / 2$ corresponds exactly to Hardy's generic quantum entanglement probability [12]. Now we seek to determine the probability $A(a, b)$ from either $A(a, b)$ or $D(a, b)$. Taking first $A(a, b)$ and setting $a=b=\phi^{5}$ as a sub-manifold probability, the joint quotient space probability is given by

$$
A\left(a=\phi^{5}, b=\phi^{5}\right)=\frac{\left(\phi^{5}\right)\left(\phi^{5}\right)}{\phi^{5}+\phi^{5}}=\phi^{5} / 2 .
$$

From $D(a, b)$ we have on the other hand the inverse value

$$
\begin{aligned}
& D(a, b)=1 / A(a, b)=2 / \phi^{5} \\
& =(2)\left(11+\phi^{5}\right)=22.18033989 .
\end{aligned}
$$

Consequently we see a clear indication of the involvement of and role played by the extra 22 "dark" dimensions which initially troubled Veneziano and his colleagues at the time. There is thus a strong and direct link between the 22 "dark" dimensions, Hardy's quantum entanglement which was obtained using orthodox quantum mechanics and which was firmly confirmed experimentally and dark matter which is strictly speaking still hypothetical but also firmly established in real measurement of accelerated expansion consistent with repulsive antigravity nature of this dark energy $[1,2,11]$. However, the situation changes completely when we realize that dark energy is nothing else but the energy of the quantum wave as we will reason later on.

\section{Revising Einstein's $E=m c^{2}$ to the Sum of $E_{1}=m c^{2} / 22$ and $E_{2}=m c^{2}(21 / 22)$}

From the above it is clear that special relativity is not quantized in any suitable form, says the Bosonic GuptaBleuler quantization and thus did not take the needed extra 22 dimensions into account nor did it have any provision for quantum entanglement. To improve special relativity its equations must intersect the equations of quantum mechanics in one way or another and a Hardy or Immirzi-type of probabilistic quantum entanglement must be planted in it [11]. Assuming the validity of Weyl scale relativity [4] and having faith in the perfect simplicity of capricious but not malicious mother nature and father time, we could reduce the task to intersecting $E=m c^{2}$ with Hardy's probability $\phi^{5}$ [12] in the form of $A=\phi^{5} / 2$ of the Veneziano model $[7,16]$. This expectation is readily fulfilled in a remarkable almost surreal way. Setting $A=\phi^{5} / 2$ as a scaling $\lambda$, we see that

$$
\lambda E \rightarrow E_{Q R}
$$

for

$$
\lambda \rightarrow \phi^{5} / 2
$$

so that

$$
E_{Q R}=\frac{\phi^{5}}{2} m c^{2}=\frac{m c^{2}}{22+k}=\frac{m c^{2}}{22.18033989} \cong \frac{m c^{2}}{22} .
$$

Remember that special relativity is based on only 1 degree of freedom elementary particle which is the photon [11] while the standard model has 12 messenger particles $[10,11,14]$, i.e. the dimension of $|S U(3)|=8$ plus $|S U(2)|=3$ and $|U(1)|=1$ where $S U(3), S U(2)$ and U(1) denote the Lie symmetry groups of the standard model [4]. Subtracting the photon then special relativity has 11 particles less. These are equal to the degrees of freedom of the standard model [11] minus one $(12-1=$ 11). Not only that but also the eleven super symmetric partners are neither known nor considered, not even hypothetically. Thus in all $2(11)=22$ needed degrees of freedom needed to minimize energy were not considered. This is how the reduction scaling $1 / 22$ could be explained. It is one explanation of many others. The end result must however be real because these are not idle theoretical discussions of academic value but rather serious measurement data [1] suggesting serious shortcomings of something which was shown here to be interpreting special relativity wrongly. Of course we have not yet found any super symmetric particles but this would not invaliddate our argument because we have many others, all leading to the same conclusions. For instance the second Betti number $b_{2}$ [13] is equal 1 for flat connected spaces just like that of special relativity. However, $b_{2}$ is exactly 22 for a K3 Kähler manifold [19,20] with 22 more 3 dimensional holes in it than in a flat connected manifold or 
a de Sitter space-time [11]. This again leads to $\lambda=b_{2}(S R) / b_{2}(K 3$ Kähler $)=1 / 22$ and a reduction in the predicted energy by $95.49 \%$ [1]. The truly surprising part of our analysis is that this shortfall in the energy density household exists in the form of dark energy which is the energy of the quantum wave [22-26]. Further careful analysis conducted over the last three years using K-theory in conjunction with non-commutative geometry, E-infinity theory and Wooden's ultimate L-theory lead to the realization that the quantum wave is an empty set in a quintic Kaluza-Klein space and is the source of non-measurable dark energy density equal $E_{2}=E(D)=\frac{5 \phi^{5}}{2} m c^{2} \cong \frac{21}{22} m c^{2}$. By contrast the measured ordinary energy $E_{1}=E(O)=\frac{\phi^{5}}{2} m c^{2} \cong \frac{m c^{2}}{22}$ is found to be the energy of the zero set also in quintic $5 D$ space-time.

\section{Kolmogorov and the 26 Strings Dimensions}

In Ref [21] Kolmogorov, who of course never worked in string theory or high energy physics is quoted on a "question of topology" where he wrote: "It seems to me that it is not difficult to prove that an $\mathrm{n}$ dimensional closed set may be embedded in a space of sufficiently large dimension and in only one way. I know how to prove this for polyhedra which is embedded in Euclidean space of dimension [21]"

$$
D=4 n+2 \text {. }
$$

Here is a faint connection to Nash's powerful embedding theorem, however it is the same formula given in Vol. II, page 371 of Green, Schwarz and Witten's classical book on string theory [15]. Thus $n$ could be considered not just a number but a dimension of the object to be embedded. For $n=0$ one finds the world sheet $D=2$. For $n=1$ we find $(4)(1)+2=6$, i.e. $D=6$ of the mass sector. For $n=6$ one finds (4)(6) $+2=26$ of Bosonic strings. Interestingly for a five dimensional object, say a quantum object with three space dimensions, one Klein-Kaluza dimension and one spin 1/2 degree of freedom, we find $(4)(5)+2=22$ dimensions. Adding the $3+1$ space and time dimension to that we retrieve the 26 dimensions again. We discussed this point only because it highlights the important role played by Euclidean embedding in high energy space-time topology and its interesting relation to the vital 22 "dark" dimensions behind the so called missing dark energy of the cosmos [1-4].

\section{Discussion}

In this section we would like to contemplate the reason why our present analysis at the simple answers which agrees with measurements for a problem which seemed to require far more complex reasoning and analysis [2763]. In addition we discuss some recent advances in our understanding of basic problems connected to fundamental questions in quantum mechanics and cosmology [33-41].

Let us start with a general observation documenting two basic shortcomings in the mathematical formulation of physical processes and the geometrical shape of the space-time in which these processes are taking place or are part of. Considering the vast mathematical literature on the continuum hypothesis and set theory, it is quite surprising that physicists do not make a proper and sharp distinction between three notions namely zero, being empty or not being there at all $[31,56,57]$. Philosophers make a distinction between being and nothingness and devoted entire monumental books to this subject, for instance "Being and Nothingness" by J. P. Sartre or even "Being and Time" by M. Heidegger [64,65] which influenced Sartre. On the other hand, at least since the founding father of set theory, G. Cantor, pure mathematicians make a definite distinction between a zero set which has only zeros in it as elements and the empty set which has no elements what so ever in it despite being a set and pure insubstantial nothingness which is not there at all as a set [31,32]. In physics on the other hand, except when it is handled by mathematicians of the calibre of Grothendieck, Attaya and Connes [10,57,58], zero means empty as well as nothing and here lies the origin of the difficulties which crept into physics and manifested itself in our understanding or rather our misunderstanding of the wave function of quantum mechanics and dark energy in cosmology to mention only two of the major problems in theoretical physics $[53,54]$. The theory presented here is more connected to reality mainly because we started from Cantor sets and fractals and went further all the way to the ultimate logic of H. Wooden [32] and defined the wave as the empty set which covers the particle modelled by the zero set. That way the negative energy of the wave reveals itself as being nothing else but the dark energy we are searching for while the measurement problem disappears altogether as a problem and becomes a natural consequent of interfering with an empty set rendering it non-empty [55-58]. Conventional particle-wave duality is seen as a kind of Hamlet to be or not to be situation. It is a particle or it is a wave depending upon the set up. Our theory presented here proves it to be slightly but importantly different because the wave is the surface (i.e. cobordism) of the particle in a five dimensional space-time. Since a particle is a zero set, its topological dimension is zero and therefore by MengerUrysohn deductive dimension theory, the surface, i.e. the wave topological dimension is minus one. That means it is the classical empty set. In other words, it is not to be or 
not to be but rather to be and not to be at the same time. This fundamental mathematical conclusion was reinforced vigorously by yet more convincing experimental results shown in a beam splitter set up that we have a quantum object which shows simultaneously in a fuzzy way both attributes of a particle and a wave [33-36]. This squares with our theory and stresses the physical reality of the wave function because an empty set is not only the beginning of mathematics but also the starting point of physics [31].

In our theory we are using a space-time geometry described not only by a topological dimension but also by a Hausdorff dimension. The basic core dimension of Einfinity is $4+\phi^{3}=4.236067977$ [22-31]. This has two consequences. First this Hausdorff dimension is a measurement for disorder, i.e. entropy. This brings our work near to that of T. Jacobson [37,38] and we may remind our readers of what was termed by some authors as the Feynman-El Naschie conjecture that gravity is the fluctuation of fractal time in space-time creating a force similar to van der Waal's force which we recognize as gravity [42]. Second, $4+\phi^{3}$ can be used for exact calculation although it has an infinite decimal expansion [22-31]. This infinity is not rejected or artificially banned from our theory but rather it is included as an important integral part of it $[41,42]$. In this context we must reiterate that fractal logic must be used as fractal counting in evaluating Higgs' experiments $[39,40]$. The graviton and the Higgs could not be counted as integer numbers for the reasons mentioned on many previous occasions [2231]. We reasoned elsewhere that the 12 messenger particles of the standard model are in fact 14 particles when counted classically using integers and crisp symmetry groups. However using fractal weight-logic and fuzzy symmetry groups we have

$\sqrt{\overline{\alpha_{0}}}=\sqrt{137.082039325}=11.708033989$ particles including the graviton and the Higgs [22-31]. Seen from a classical view point this seems incomprehensible but fractally this makes a great deal of sense and explains why the Higgs mass and spin were not found in a watertight conclusive way in the recent CERN experiments. It is part of the nature of the Higgs and the Higgs field may also be an approximate way of looking at our empty setzero set quantum space-time.

Finally we address the global nature of the Cantorian geometry used to arrive at our present conclusions and that $E(D)=(21 / 22) m c^{2}$ is not only $95.5 \%$ of the total energy of the universe but also that it is a negative energy creating a negative repulsive gravity leading to the observed acceleration rather an deceleration of the expansion of the cosmos. Globally our fractal-Cantorian spacetime is a material space like the space of the theory of elasticity, only more sophisticated $[60,61]$. The nearest known theory to that would be Cartan theory which in- cludes torsion tensors or even better, the theory of polar media of the French electrical engineering brothers Cosserat [62].

In such material space, anticlastic curvature is a natural outcome of any induced curvature [61]. Thus as in the simple elementary demonstration with a long cylindrically rolled paper sheet of Figure 14, Ref. [24], we have anticlastic curvature at the extremity of the cylinder. In the holographic projection of the Penrose-Klein modular curve hyperbolic universe, the extremity is a ramified circular edge encircling the curve. That is where negative energy produces antigravity and antigravity in the form of anticlastic curvature produces negative energy in a circulatory logic blurring the distinction between cause and effect. The situation may be made analogous to the horizon of a black hole where Hawking's vacuum fluctuation produces the negative energy of the vacuum [4245]. As things stand now we may naively define classicality or Newtonian mechanics as an averaging compromise between the wave aspect and the particle aspect so that the energy is half of that given by Einstein's equation

$E_{N}=\frac{1}{2}(E(O)+E(D))=E=\frac{1}{2} m(c \rightarrow v)^{2}$. On the other hand Einstein's energy could be thought of equally naively as a perfectly Newtonian kinetic energy with a single character fault of having a constant speed $v=c=$ constant from the beginning to the end of the time interval of the motion of a particle with mass $m$ so that $v=c=$ constant can be taken out of the integration and one finds that $E=m(v=c)^{2}=m c^{2}$. It is the subtlety of this situation which may be the cause of many misunderstandings of Einstein's relativity as well as Newtonian mechanics in equal measures.

\section{Conclusions}

In this paper we use chaos theory and fractal geometry to utilize a formal correspondence between the Veneziano dual resonance model and quotient non-commutative spaces [10]. Veneziano's model makes sense only in 26 dimensions [15]. Building a bridge between this model and special relativity we see that $E=m c^{2}$ includes only 4 dimensions. This is 22 less than what the strong interactions need in the Veneziano model in order to get rid of the negative norms. This is also approximately the scaling needed to elevate $E=m c^{2}$ from a formula of special relativity to $E=(1 / 22.18033989) m c^{2}$, which is a formula taking on board things like generic Hardy's quantum entanglement [12], entire spectrum of the elementary messenger particles of minimally super symmetrically extended standard model as well as 22 extra dimensions which we call "dark dimensions" [22-29]. This is because if dark energy really exists and we do 
believe that it exists being the absolute value of the negative energy of the quantum wave, then dark dimensions and fractal voids are the place where it can screen its existence. Mathematically these screened areas and voids in space-time are the empty sets or the fat fractal contraparts of a KAM space-time [23,26]. We cannot answer all these questions fully but at least the prediction of our quantum relativity formula [14] $E \cong m c^{2} / 22$ is in almost perfect agreement with cosmological measurements [1]. The present result is in any event real and not speculative in any sense because it starts from a conventional picture and gives results in excellent agreement with actual highly accurate measurements $[1,27,30]$.

Taken as a whole, the present results as well as the results and conclusions of previous publications [22-30] suggest the following picture based on the stringent mathematical logic of transfinite set theory [31,32]. Spacetime is the origin of both the quantum wave and the quantum particle. The first is essentially a five dimensional empty set [31]. The second is also five dimensional but a zero set [27-31]. The empty set is the cobordism of the zero set and may be likened to a Dirac's hole with negative energy. Since interfering with an empty set, for instance at measurement, renders it nonempty, wave collapse is a natural result of any quantum measurement. This is the only mathematically well defined meaning of the quantum wave collapse on measurement [26,31]. That is why we cannot detect the energy of the quantum wave and quantum mechanics inaccurately concluded that the quantum wave is devoid of any energy [11,22-31]. In reality a quantum wave is devoid of any ordinary positive energy but it has a considerable amount of negative energy. This fact was discovered experimentally not in any high energy laboratory but in a completely different field using the entire cosmos as an experimental set up [1-4]. There are other relatively recent experimental and theoretical works which support our conclusion in broad general terms [33-40]. For instance, recent experiments with beam splitters shows neither quantum particle behaviour nor quantum wave behaviour but a little of both together [33-36]. This agrees completely with our mathematical definition which says that the wave is just the out surface (cobordism) of the particle which means there is no way to really separate the one from the other and that the wave is just as real as the particle [31]. Furthermore, our picture of the anticlastic space-time curvature produced by the dark energy of the wave at the edge of the universe causing negative gravity agrees in principle with some features of the work of T. Jacobson [37,38]. In addition L. Krauss $[39,40]$ and others connecting the Higgs to dark energy is not that far short from our concept of a KAM space-time which resembles a Higgs field [22-30]. Finally our theory can easily embrace ideas about the importance of Basian probability [41]. Never the less, the theory of probability is not the best way to start when you need to define a particle and a wave. It is set theory and fractal Cantor set theory [54] with its zero and empty set which must be our first step as we did in the present work and earlier publications [38,39].

\section{REFERENCES}

[1] L. Amendola and S. Tsujikawa, "Dark Energy, Theory and Observations," Cambridge University Press, Cambridge, 2010.

[2] B. Carr, "Universe or Multiverse?" Cambridge University Press, Cambridge, 2010.

[3] Y. Baryshev and P. Teerikorpi, "Discovery of Cosmic Fractals," World Scientific, Singapore, 2011.

[4] L. Nottale, "Scale Relativity," Imperial College Press, London, 2011.

[5] G. Barenblatt, "Scaling," Cambridge University Press, Cambridge, 2003. http://dx.doi.org/10.1017/CBO9780511814921

[6] J. Mageuijo and L. Smolin, "Lorentz Invariance with an Invariant Energy Scale," arXiv: hep-th/0112090V2, 18 December 2001.

[7] G. Veneziano, "Ward Identities in Dual String Theories," Physics Letters B, Vol. 167, No. 4, 1986, pp. 388-392.

[8] Y. Nambu, "Quark Model and the Factorization of Veneziano Amplitude, In: R. Choud, Ed., Symmetries and Quark Models, Gordon and Breach, New York, 1970, pp. 269-278.

[9] V. Vladimirov. I. Valovich and E. Zelenov, "P-Adic Analysis and Mathematical Physics," World Scientific, Singapore, 1994. http://dx.doi.org/10.1142/1581

[10] A. Connes, "Non-Commutative Geometry," Academic Press, San Diego, 1994.

[11] R. Penrose, "The Road to Reality," Jonathan Cape, London, 2004.

[12] L. Hardy, "Non-Locality of Two Particles without Inequalities for Almost All Entangled States," Physical Review Letters, Vol. 71, No. 11, 1993, pp. 1665-1668. http://dx.doi.org/10.1103/PhysRevLett.71.1665

[13] C. Nash and S. Sen, "Topology and Geometry for Physicists," Academic Press, San Diego, 1983.

[14] I. Buchbinder, S. Odintsov and I. Shapiro, "Effective Action in Quantum Gravity," Institute of Physics Publishing, Bristol, 1992.

[15] M. Green, J. Schwarz and E. Witten, "Superstring Theory," Cambridge University Press, Cambridge, 1987.

[16] M. Kaku, "Introduction to Superstrings and M-Theory," Springer, New York, 1999.

http://dx.doi.org/10.1007/978-1-4612-0543-2

[17] J.-H. He, "Hilbert Cube Model for Fractal Space-Time," Chaos, Solitons \& Fractals, Vol. 42, No. 5, 2009, pp. 2754-2759. http://dx.doi.org/10.1016/j.chaos.2009.03.182

[18] J.-H. He, "Twenty Six Dimensional Polytope and High 
Energy Spacetime Physics," Chaos, Solitons \& Fractals, Vol. 33, No. 1, 2007, pp. 5-13.

http://dx.doi.org/10.1016/j.chaos.2006.10.048

[19] D. Joyce, "Compact Manifold with Special Holonom," Oxford Press, Oxford, 2003.

[20] T. Hübsch, "Calabi-Yau Manifolds," World Scientific, Singapore, 1994.

[21] E. Charpentier, A. Lesne and N. Nikolski, "Kolmogorov's Heritage in Mathematics," Springer, Berlin, 2007.

[22] M. S. El Naschie, "Quantum Entanglement: Where Dark Energy and Negative Gravity plus Accelerated Expansion of the Universe Comes From," Journal of Quantum Information Science, Vol. 3, No. 2, 2013, pp. 57-77. http://dx.doi.org/10.4236/jqis.2013.32011

[23] M. S. El Naschie, "The Missing Dark Energy of the Cosmos From Light Cone Topological Velocity and Scaling the Planck Scale," Open Journal of Microphysics, Vol. 3, No. 3, 2013, pp. 64-70. http://dx.doi.org/10.4236/ojm.2013.33012

[24] M. S. El Naschie and A. Helal, "Dark Energy Explained via the Hawking-Hartle Quantum Wave and the Topology of Cosmic Crystallography," International Journal of Astronomy and Astrophysics, Vol. 3, No. 3, 2013, pp. 318343.

[25] M. S. El Naschie, "The Quantum Gravity Immirzi Parameter-A General Physical and Topological Interpretation," Gravitation and Cosmology, Vol. 19, No. 3, 2013, pp. 151-155. http://dx.doi.org/10.1134/S0202289313030031

[26] M. S. El Naschie, "What Is the Missing Dark Energy in a Nutshell and the Hawking-Hartle Quantum Wave Collapse," International Journal of Astronomy and Astrophysics, Vol. 3, No. 3, 2013, pp. 205-211. http://dx.doi.org/10.4236/ijaa.2013.33024

[27] L. Marek-Crnjac, "Modification of Einstein's $E=m c^{2}$ to $E=m c^{2} / 22$," American Journal of Modern Physics, Vol. 2, No. 5, 2013, pp. 255-263.

[28] M. S. El Naschie, "A Resolution of the Cosmic Dark Energy via a Quantum Entanglement Relativity Theory," Journal of Quantum Information Science, Vol. 3, No. 1, 2013, pp. 23-26. http://dx.doi.org/10.4236/jqis.2013.31006

[29] M. S. El Naschie, "Dark Energy from Kaluza-Klein Spacetime and Noether's Theorem via Lagrangian Multiplier Method," Journal of Modern Physics, Vol. 4, No. 6, 2013, pp. 757-760. http://dx.doi.org/10.4236/jmp.2013.46103

[30] M. S. El Naschie, "Determining the Missing Dark Energy of the Cosmos from a Light Cone Exact Relativistic Analysis," Journal of Modern Physics, Vol. 2, No. 2, 2013, pp. 18-23.

[31] M. S. El Naschie, "Towards a General Transfinite Set Theory for Quantum Mechanics," Fractal Space-Time and Non-Commutative Geometry in High Energy Physics, Vol. 2, No. 2, 2012, pp. 135-142.

[32] R. Elwes, "Ultimate Logic," New Scientist, Vol. 211, No. 2823, 2011, pp. 30-33.

http://dx.doi.org/10.1016/S0262-4079(11)61838-1
[33] V. Jacques, et al., "Delayed-Choice Test of Quantum Complementarity with Interfering Single Photons," Physical Review Letters, Vol. 100, No. 22, 2008, Article ID: 220402.

http://dx.doi.org/10.1103/PhysRevLett.100.220402

[34] L. Li, N. L. Liu and Z. X. Yu, "Duality Relations in a Two Path Interferometer with an Asymmetric Beam Splitter," Physical Review A, Vol. 85, No. 5, 2012, Article ID: 054101.

http://dx.doi.org/10.1103/PhysRevA.85.054101

[35] M. F. Schriber, "Another Step Back for Wave-Particle Duality," Physics, Vol. 4, No. 102, 2011, Article ID: 230406.

[36] J.-S. Tang et al., "Revisiting Bohr's Principle of Complementarity Using a Quantum Device," arXiv: 1204.5304V1[quant-ph], 24 April 2012.

[37] T. Jacobson, et al., "Increase of Black Hole Entropy in Higher Curvature Gravity,” arXiv: gr-qc/9503020V1, 11 March 1995.

[38] V. Vedral, "In from the Cold," New Scientist, Vol. 216, No. 2886, 2012, pp. 33-37.

[39] L. M. Krauss, "A Higgs-Saw Mechanism as a Source of Dark Energy," arXiv:1306.3239V1[hep-ph], 13 June 2013.

[40] L. Grossman, "Dark Energy May Spring from the Higgs Boson," New Scientist, Vol. 219, No. 2931, 2013, p. 11. http://dx.doi.org/10.1016/S0262-4079(13)62067-9

[41] D. Mermin, "Quantum Mechanics: Fixing the Shifty Split," Physics Today, Vol. 65, No. 7, 2012, pp. 8-10. http://dx.doi.org/10.1063/PT.3.1618

[42] M. S. El Naschie, "A Note on Quantum Gravity and Cantorian Spacetime," Chaos, Solitons \& Fractal, Vol. 8, No. 1, 1997, pp. 131-133. http://dx.doi.org/10.1016/S0960-0779(96)00128-2

[43] M. S. El Naschie, "Complex Vacuum Fluctuation as a Chaotic 'Limit' Set of Any Kleinian Group Transformation and the Mass Spectrum of High Energy Particle Physics via Spontaneous Self-Organization," Chaos, Solitons \& Fractals, Vol. 17, No. 4, 2003, pp. 631-638. http://dx.doi.org/10.1016/S0960-0779(02)00630-6

[44] M. S. El Naschie, "VAK, Vacuum Fluctuation and the Mass Spectrum of High Energy Particle Physics," Chaos, Solitons \& Fractals, Vol. 17, No. 4, 2003, pp. 797-807. http://dx.doi.org/10.1016/S0960-0779(02)00684-7

[45] M. S. El Naschie, "The VAK of Vacuum Fluctuation, Spontaneous Self-Organization and Complexity Theory Interpretation of High Energy Particle Physics and the Mass Spectrum," Chaos, Solitons \& Fractals, Vol. 18, No. 2, 2003, pp. 401-420. http://dx.doi.org/10.1016/S0960-0779(03)00098-5

[46] J.-H. He, "A Note on Elementary Cobordism and Negative Space," International Journal of Nonlinear Sciences and Numerical Simulation, Vol. 11, No. 12, 2010, pp. 1093-1095.

[47] M. S. El Naschie, “Average Symmetry, Stability and Ergodicity of Multidimensional Cantor Sets," Il Nuovo Cimento, Vol. 109, No. 2, 1994, pp. 149-157.

http://dx.doi.org/10.1007/BF02727425 
[48] M. S. El Naschie, "Mathematical Foundations of E-Infinity via Coxeter and Reflection Groups," Chaos, Solitons \& Fractals, Vol. 37, No. 5, 2008, pp. 1267-1268. http://dx.doi.org/10.1016/j.chaos.2008.02.001

[49] M. S. El Naschie, "Removing Spurious Non-Linearity in the Structure of Micro-Space-Time and Quantum Field Renormalization," Chaos, Solitons \& Fractals, Vol. 37, No. 1, 2008, pp. 60-64. http://dx.doi.org/10.1016/j.chaos.2007.10.005

[50] M. S. El Naschie, "On 't Hooft Dimensional Regularization in E-Infinity Space," Chaos, Solitons \& Fractals, Vol. 12, No. 5, 2001, pp. 851-858. http://dx.doi.org/10.1016/S0960-0779(00)00138-7

[51] O. E. Rössler, et al., "Hubble Expansion in Static SpaceTime," Chaos, Solitons \& Fractals, Vol. 33, No. 3, 2007 , pp. 770-775. http://dx.doi.org/10.1016/j.chaos.2006.06.046

[52] M. Pusey, J. Barrett and T. Randolph, "On the Reality of Quantum State," Nature Physics, Vol. 8, June 2012, pp. 475-478.

[53] M. S. El Naschie, "Mohamed El Naschie Answers a Few Questions about This Month's Emerging Research Front in the Field of Physics," 2004.

http://esi-topics.com/erf/2004/october04-MohamedElNas chie.html

[54] M. S. El Naschie, "This Month's New Hot Paper in the Field of Engineering: On a Fuzzy Kähler-Like Manifold Which Is Consistent with the Two Slit Experiment," International Journal of Nonlinear Sciences and Numerical Simulation, Vol. 6, No. 2, 2005, pp. 95-98. http://esi-topics.com/nhp/2006/september-06-MohamedEl Naschie.html

[55] M. Persinger and C. Lavellee, "Theoretical and Experimental Evidence of Macroscopic Entanglement between Human Brain Activity and Photon Emission," Journal of Consciousness Exploration \& Research, Vol. 1, No. 7, 2010, pp. 785-807.

[56] M. S. El Naschie, "COBE Satellite Measurement, Can- torian Space and Cosmic Strings," Chaos, Solitons \& Fractals, Vol. 8, No. 5, 1977, pp. 847-850. http://dx.doi.org/10.1016/S0960-0779(97)00084-2

[57] L. Marek-Crnjac, "The Physics of Empty Sets and the Quantum," Nonlinear Science Letters B, Vol. 1, No. 1, 2011, pp. 13-14.

[58] J.-H. He, "The Importance of the Empty Set Underpinning the Foundation of Quantum Physics," Nonlinear Science Letters B, Vol. 1, No. 1, 2011, pp. 6-7.

[59] M. S. El Naschie, "Penrose Universe and Cantorian Spacetime as a Model for Noncommutative Quantum Geometry," Chaos, Solitons \& Fractals, Vol. 9, No. 6, 1998, pp. 931-933. http://dx.doi.org/10.1016/S0960-0779(98)00077-0

[60] M. S. El Naschie, "Stress, Stability and Chaos in Structural Engineering," McGraw Hill, London, 1990.

[61] D. Horrockos and W. Johnson, "On Anticlastic Curvature with Special Reference to Plastic Bending," The International Journal of Mechanical Sciences, Vol. 9, No. 12, 1967, pp. 835-861. http://dx.doi.org/10.1016/0020-7403(67)90011-2

[62] E. Cosserat and F. Cosserat, "Theorie des Corps Deformables,” Lavoisier S.A.S., Paris, 1909.

[63] M. S. El Naschie, “A Fractal Menger Sponge Spacetime Proposal to Reconcile Measurements and Theoretical Predictions of Cosmic Dark Energy," International Journal of Modern Nonlinear Theory and Application, Vol. 2, No. 2, 2013, pp. 107-121. http://dx.doi.org/10.4236/ijmnta.2013.22014

[64] M. S. El Naschie, "On the Philosophy of Being and Nothingness in Fundamental Physics," Nonlinear Science Letters A, Vol. 2, No. 1, 2011, pp. 5-6.

[65] M. S. El Naschie, "On the Mathematical Philosophy of Being and Nothingness in Quantum Physics," Fractal Space-Time \& Non-Commutative Geometry in Quantum and High Energy Physics, Vol. 2, No. 2, 2012, pp. 103106. 